\title{
The effects of aggressive vs. conservative phototherapy on the brainstem auditory evoked responses of extremely-low-birth-weight infants
}

\author{
Robert E. Lasky, ${ }^{1,2}$, Michael W. Church ${ }^{3}$, Mark S. Orlando ${ }^{4}$, Brenda H. Morris ${ }^{2}$, Nehal A. Parikh², Jon E. Tyson 1,2, \\ Georgia E. McDavid ${ }^{1,2}$, William Oh ${ }^{5}$, David K. Stevenson ${ }^{6}$, Krisa P. Van Meurs ${ }^{6}$, Ronnie Guillet ${ }^{7}$ and Dale L. Phelps ${ }^{7}$
}

INTRODUCTION: This study was a two-center, stratified, parallel-group randomized trial comparing the effects of aggressive vs. conservative phototherapy on brainstem auditory evoked response (BAER) latencies in infants with extremely low birth weight (ELBW, $\leq 1,000 \mathrm{~g})$.

RESULTS: BAER latencies of $751-1,000 \mathrm{~g}$ birth-weight infants were shorter by $0.37 \mathrm{~ms}(95 \%$ confidence interval $(\mathrm{Cl})=0.02,0.73$ ) for wave $\mathrm{V}, 0.39 \mathrm{~ms}(0.08,0.70)$ for wave $\mathrm{III}$, and $0.33 \mathrm{~ms}(0.01,0.65)$ for wave I after aggressive phototherapy at one center. Interwave intervals did not differ significantly. Similar nonsignificant trends were recorded for 501-750 g birth-weight infants. At the other participating center, no significant differences were recorded, cautioning against overgeneralizing these results.

DISCUSSION: The effects of bilirubin on the auditory pathway in ELBW infants depend on a complex interaction of bilirubin exposure, newborn characteristics, and clinical management.

METHODS: Aggressive phototherapy was initiated sooner and continued at lower bilirubin levels than conservative phototherapy. A total of 174 ELBW infants were enrolled in the study; 111 infants were successfully tested at 35 weeks postmenstrual age (PMA); 57 died; and 6 were not successfully tested.

$\mathbf{T}$ wo large trials have evaluated whether phototherapy for newborns with elevated bilirubin levels reduces the risk of adverse neurodevelopmental outcomes. There was no improvement in neurodevelopmental outcomes 6 years after phototherapy (1) in a trial conducted by Brown and colleagues (2). The National Institutes of Health and Eunice Kennedy Shriver National Institute of Child Health and Human Development (NICHD) Neonatal Research Network trial evaluated the effects of aggressive vs. conservative phototherapy in extremely-lowbirth-weight $(\leq 1,000 \mathrm{~g}, \mathrm{ELBW})$ infants (3). Death rate did not differ with the two treatments (relative risk $(\mathrm{RR})=1.05,95 \%$ confidence interval $(\mathrm{CI})=0.90,1.22$ ); however, neurodevelopmental impairment was significantly reduced among survivors with aggressive phototherapy $(\mathrm{RR}=0.86,95 \% \mathrm{CI}=0.74,0.99)$ including better performance on the Bayley Mental Developmental Index and less hearing loss and athetosis.

Bilirubin neurotoxicity prolongs neuronal transmission in the auditory brainstem as measured by brainstem auditory evoked responses (BAERs) (4). We recorded BAERs in infants with birth weights of $501-750 \mathrm{~g}$ or $751-1,000 \mathrm{~g}$ who were enrolled in two of the centers participating in the NICHD Neonatal Research Network trial.

The primary hypothesis tested was as follows:

1. Aggressive phototherapy reduces neuronal transmission times in ELBW infants at 35 weeks postmenstrual age (PMA) as measured by BAER wave $\mathrm{V}$ latency.

Secondary hypotheses tested were as follows:

1. Aggressive phototherapy reduces other measures of neuronal transmission times in ELBW infants at 35 weeks PMA as measured by the latencies of BAER waves I and III and BAER interwave intervals (V-I, III-I, V-IV, IV-III, II-I).

2. Higher total serum bilirubin (TSB) levels in the first 2 weeks after birth in ELBW infants are associated with prolonged neuronal transmission times at 35 weeks PMA as measured by BAER latencies.

Tests of the primary and secondary hypotheses included whether treatment effects differ by center and birth-weight stratum.

\section{RESULTS}

\section{Study Sample}

Figure 1 presents the study cohort. Six of the 117 (5.1\%) eligible study infants who were alive at 35 weeks PMA were not successfully tested; 4/36 (11.1\%) were from center A and 2/81 (2.5\%) were from center B. Five infants were in the aggressive phototherapy treatment arm (three at center A and two at center B). Four infants (two from each site), including an infant in the

${ }^{1}$ Center for Clinical Research and Evidence-Based Medicine, University of Texas Medical School at Houston, Houston, Texas; ${ }^{2}$ Department of Obstetrics and Gynecology, Wayne State University School of Medicine, Detroit, Michigan; ${ }^{3}$ Department of Otolaryngology, University of Rochester School of Medicine and Dentistry, Rochester, New York; ${ }^{4}$ Department of Pediatrics, University of Texas Medical School at Houston, Houston, Texas; ${ }^{5}$ Department of Pediatrics, Women \& Infants Hospital, Brown University, Providence, Rhode Island; ${ }^{6}$ Department of Pediatrics, Division of Neonatal and Developmental Medicine, Stanford University School of Medicine and Lucile Packard Children's Hospital, Palo Alto, California; 'Department of Pediatrics, University of Rochester School of Medicine and Dentistry, Rochester, New York. Correspondence: Robert E. Lasky

(Robert.E.Lasky@uth.tmc.edu) 


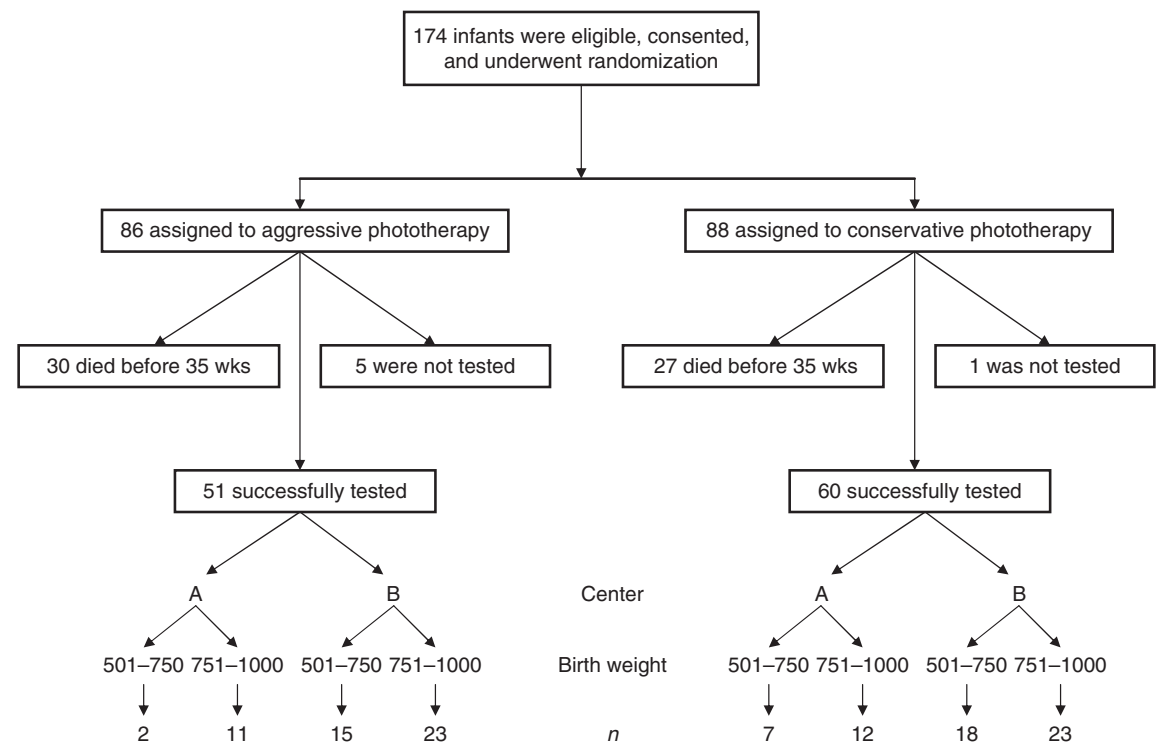

Figure 1. The number of study infants who were eligible, consented, randomized, died before the target test age (35 weeks postmenstrual age), and successfully tested.

conservative phototherapy treatment arm, were discharged or transferred prior to their test date without informing the personnel doing the BAER testing. An administrative error and unsuccessful testing due to poor recording conditions after two attempts accounted for the other two missed tests.

Of the 111 eligible infants successfully tested according to protocol, the median age at testing was 35 weeks and 1 day PMA (interquartile range $=3$ days); 106 (95.5\%) infants were tested within 1 week of 35 weeks. Three infants were tested 14 , 12 , and 10 days prior to 35 weeks because they were going to be transferred to another hospital. The other two infants were retested at 36 weeks 1 day because of poor recording conditions at 35 weeks.

Demographic and baseline characteristics of the successfully tested study infants are listed in Tables 1 and 2. There were few imbalances in the baseline variables across the two treatment conditions within the two centers. Gender was somewhat of an exception. Males were more prevalent in the conservative $(11 / 19=58 \%)$ compared to the aggressive $(4 / 13=31 \%)$ phototherapy treatment conditions at center $\mathrm{A}$. Males were more equally distributed in the conservative $(18 / 41=44 \%)$ and aggressive $(19 / 38=50 \%)$ conditions at center $B$.

There were center differences in demographic and baseline characteristics (Tables 1 and 2). Center A infants were mostly black (88\%), with a small percentage $(12 \%)$ of white infants. In contrast, $44 \%$ of center B infants were black, $30 \%$ were white, $24 \%$ were Hispanic, and one was Asian. Center A infants were significantly older at birth $(27.1 \pm 0.4$ weeks vs. $26.2 \pm 0.2$ weeks), less likely to be outborn ( $6 \%$ vs. $25 \%)$, more likely to be ventilated ( $81 \%$ vs. $62 \%$ ), and less likely to be on continuous positive airway pressure at $24 \mathrm{~h}(0 \%$ vs. $34 \%)$.

\section{Effectiveness of the Intervention}

Table 3 presents bilirubin exposure and phototherapy treatment duration means and 95\% CIs. As expected from the study design, infants in the conservative phototherapy condition had significantly higher average TSB levels for the first 14 days after initiating phototherapy $(P<0.001)$, higher peak TSB levels $(P<0.001)$, and shorter duration of phototherapy $(P<0.001)$ than infants in the aggressive phototherapy treatment arm. The 751-1,000 g birth-weight infants had significantly higher average TSB levels for the first 14 days after initiating phototherapy $(P=0.002)$ and higher peak TSB levels $(P=0.002)$ than the $501-750 \mathrm{~g}$ birth-weight infants $(P<0.001)$, although the latter result was moderated by treatment condition and center (i.e., there was a phototherapy by center by birth-weight stratum interaction, $P<$ $0.001)$. Average TSB levels for the first 14 days after initiating phototherapy $(P<0.001)$ and peak TSB levels $(P<0.001)$ were significantly higher at center $\mathrm{A}$ than at center $\mathrm{B}$. The duration of phototherapy in both treatment conditions was significantly longer at center A $(P<0.001)$.

\section{Reliability of the BAER Scoring}

The two scorers agreed that waves III and V from each ear were present for nearly the entire sample $(95.5-96.4 \%$ of the 111 infants recorded). Those measurements were scored within $0.2 \mathrm{~ms}$ of each other $89.6 \%-94.4 \%$ of recordings. Most of the few times that one scorer scored wave III or V as absent, the other did as well (4/4 times for the left ear and 3/5 times for the right ear for wave III and 4/5 times for both ears for wave V).

Wave I was the only measurement scored more reliably at one site than the other (100\% agreement that wave I was present for both ears at center A vs. $51 \%$ for the left and $48 \%$ for the right ear at center $\mathrm{B})$. The noninverting electrode was placed higher on the head at center B than center A to avoid CPAP tubes and nasal cannulae negatively affecting the recording of wave I. As a consequence, wave I was less prominent and often confused with wave II. For nearly $40 \%$ of the recordings, either both scorers or at least one scored II and also IV as absent. 
Table 1. Demographic and baseline characteristics of the study infants from center $A$

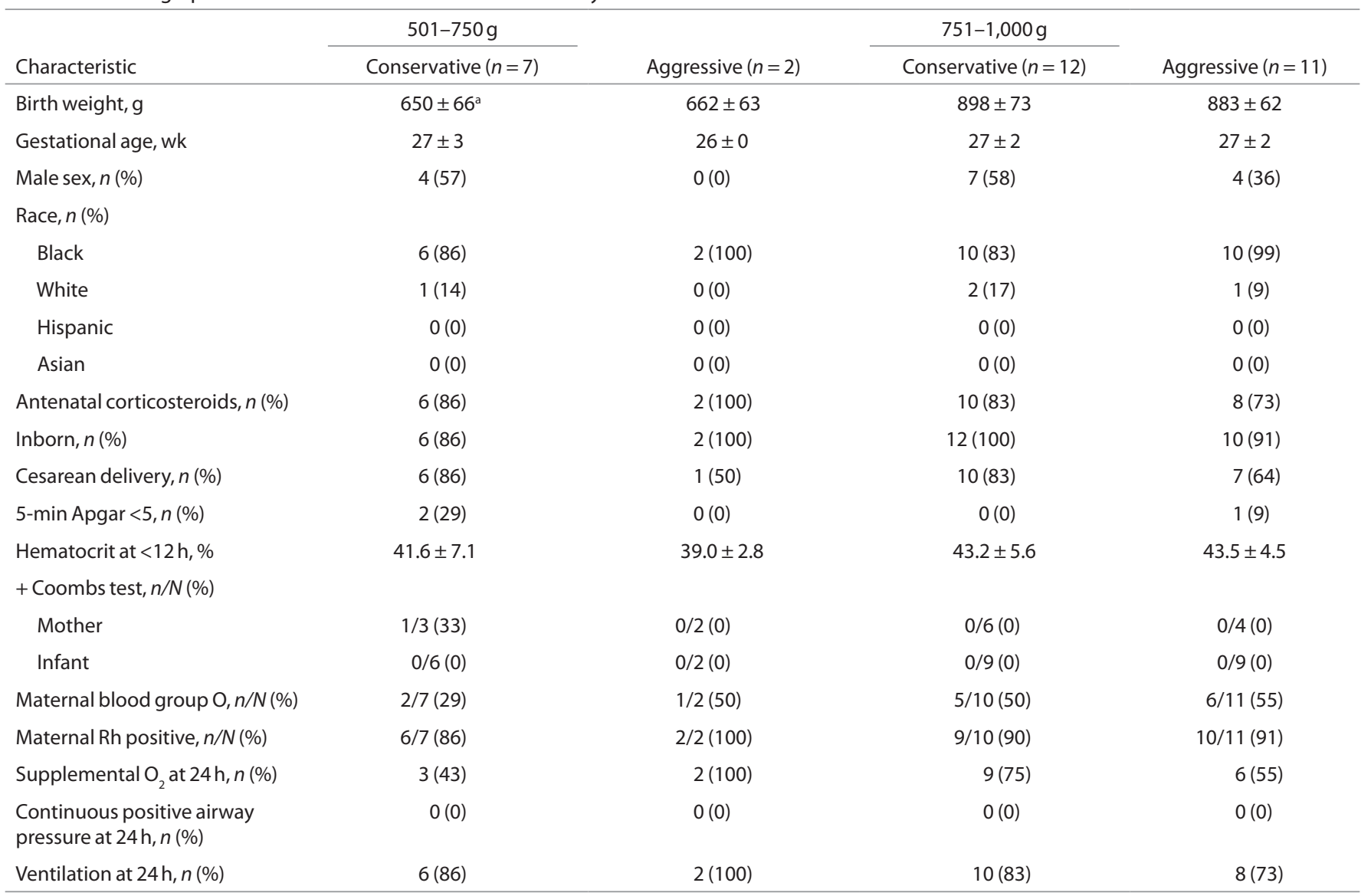

aAll continuous variables are described by the mean \pm SD.

\section{Treatment Effects on the BAER}

Table 4 presents mean (95\% CI) differences in BAER latencies calculated from regression models for infants in the aggressive and conservative treatment conditions by center and birthweight stratum. The results presented in Table 4 are averaged over the two ears.

The 751-1,000 g birth-weight center A infants in the aggressive compared to the conservative phototherapy treatment condition had significantly shorter wave $\mathrm{V}$ latencies and also significantly shorter wave I and III latencies. Similar nonsignificant trends were recorded for the 501-750 g birth-weight center A infants. The small number of infants in the 501-750 g birth-weight stratum at center A limits the interpretation of those results. At center $\mathrm{B}$, there were no significant differences in BAER latencies between treatment conditions.

There was little support for different phototherapy treatment effects in 501-750 g and 751-1,000 g infants. None of the threeway or two-way phototherapy treatment by birth-weight stratum interactions were significant. In contrast, there was some statistical support for different phototherapy treatment effects in the two centers (i.e., treatment by center interactions). Center differences in the phototherapy treatment effects were robust for wave III latencies $(P=0.032)$ but not for wave $\mathrm{V}(P=0.239)$ and wave I $(P=0.174)$ latencies. Given the small sample size, nonsignificant interactions are not surprising.
Aggressive phototherapy treatment did not reduce brainstem transmission times as measured by the V-I, V-III, and III-I interwave latencies (see Table 4). These results are consistent with a peripheral (i.e., the distal auditory nerve and/ or the inner, middle, or outer ears) and not a brainstem site of lesion. Axonal vs. synaptic transmission was not significantly different in aggressive and conservative phototherapytreated infants, with one exception. There was a significant phototherapy treatment by birth-weight stratum interaction $(P=0.036)$ for the IV-III interwave latencies, indicating that aggressive phototherapy was associated with faster axonal transmission in the 501-750 g birth-weight infants. The last row of Table 4 presents the II-I interwave latencies for the 94 infants whose latencies could be scored in at least one ear. The II-I latency interval also measures axonal transmission (5). There was no support for phototherapy treatment differences for wave II-I interwave latencies, suggesting that the phototherapy treatment effect for the 501-750 g birthweight infants for the IV-III interwave latencies may be a chance result.

Adjusting for the age at testing and each of the baseline and demographic variables listed in Table 3 did not significantly alter the phototherapy treatment effects reported in Table 4 . In addition, adjusting for possible mediators having their onset after the initiation of phototherapy but prior to the 35-week assessment 


\section{Articles | Lasky et al.}

Table 2. Demographic and baseline characteristics of the study infants from center $B$

\begin{tabular}{|c|c|c|c|c|}
\hline \multirow[b]{2}{*}{ Characteristic } & \multicolumn{2}{|c|}{$501-750 \mathrm{~g}$} & \multicolumn{2}{|c|}{$751-1,000 \mathrm{~g}$} \\
\hline & Conservative $(n=18)$ & Aggressive $(n=15)$ & Conservative $(n=23)$ & Aggressive $(n=23)$ \\
\hline Birth weight, $g$ & $630 \pm 68^{\mathrm{a}}$ & $647 \pm 69$ & $884 \pm 68$ & $890 \pm 74$ \\
\hline Male sex, $n(\%)$ & $7(39)$ & $6(40)$ & $11(48)$ & $13(57)$ \\
\hline \multicolumn{5}{|l|}{ Race, $n(\%)$} \\
\hline White & $4(22)$ & $3(20)$ & $7(30)$ & $10(43)$ \\
\hline Hispanic & $5(28)$ & $4(27)$ & $6(26)$ & $4(17)$ \\
\hline Asian & $1(6)$ & $0(0)$ & $0(0)$ & $0(0)$ \\
\hline Antenatal corticosteroids, $n / N(\%)$ & $12 / 18(67)$ & $9 / 14(64)$ & $15 / 23(65)$ & $18 / 23(78)$ \\
\hline Inborn, $n(\%)$ & $12(67)$ & $13(87)$ & $16(70)$ & $18(78)$ \\
\hline \multicolumn{5}{|l|}{ + Coombs test, $n / N(\%)$} \\
\hline Mother & $0 / 15(0)$ & $1 / 15(7)$ & $2 / 22(9)$ & $0 / 22(0)$ \\
\hline Infant & $0 / 18(0)$ & $1 / 15(7)$ & $0 / 23(0)$ & $0 / 23(0)$ \\
\hline Maternal blood group O, $n / N(\%)$ & $6 / 16(38)$ & $9 / 15(60)$ & $8 / 22(36)$ & $10 / 23(43)$ \\
\hline Maternal Rh positive, $n / N(\%)$ & $16 / 16(100)$ & $14 / 15(93)$ & $18 / 22(82)$ & $21 / 23(91)$ \\
\hline Supplemental $\mathrm{O}_{2}$ at $24 \mathrm{~h}, n(\%)$ & $14(78)$ & $8(53)$ & $14(61)$ & $14(61)$ \\
\hline $\begin{array}{l}\text { Continuous positive airway } \\
\text { pressure at } 24 \mathrm{~h}, n(\%)\end{array}$ & $3(17)$ & $2(13)$ & $13(57)$ & $9(39)$ \\
\hline Ventilation at $24 \mathrm{~h}, n(\%)$ & $14(78)$ & $13(87)$ & $10(43)$ & $12(52)$ \\
\hline
\end{tabular}

${ }^{a}$ All continuous variables are described by the mean \pm SD

Table 3. Bilirubin measurements as a function of phototherapy condition, center, and birth-weight stratum

\begin{tabular}{|c|c|c|c|c|}
\hline \multirow[b]{2}{*}{ Bilirubin measurements } & $501-750 \mathrm{~g}$ & \multirow[b]{2}{*}{ Aggressive } & $751-1,000 \mathrm{~g}$ & \multirow[b]{2}{*}{ Aggressive } \\
\hline & Conservative & & Conservative & \\
\hline \multicolumn{5}{|l|}{ TSB 14-day average (mg/dl) } \\
\hline Center A, mean $(95 \% \mathrm{Cl})$ & $6.5(5.6,7.3)$ & $5.6(4.0,7.2)$ & $8.2(7.6,8.9)$ & $6.0(5.3,6.7)$ \\
\hline Center B, mean (95\% Cl) & $4.9(4.4,5.4)$ & $3.7(3.2,4.3)$ & $5.9(5.4,6.4)$ & $4.5(4.0,4.9)$ \\
\hline \multicolumn{5}{|l|}{ TSB peak value (mg/dl) } \\
\hline Center A, mean $(95 \% \mathrm{Cl})$ & $10.0(8.8,11.2)$ & $13.4(11.1,15.7)$ & $12.5(11.6,13.5)$ & $8.9(7.9,9.9)$ \\
\hline Center B, mean (95\% Cl) & $9.0(8.3,9.8)$ & $5.9(5.0,6.7)$ & $9.6(8.9,10.3)$ & $6.5(5.9,7.2)$ \\
\hline \multicolumn{5}{|l|}{ Age at peak value (h) } \\
\hline Center A, mean $(95 \% \mathrm{Cl})$ & $59.8(13.7,105.8)$ & $92.2(6.1,178.3)$ & $102.5(67.4,137.7)$ & $115.2(78.4,151.9)$ \\
\hline Center B, mean $(95 \% \mathrm{Cl})$ & $74.1(45.4,102.8)$ & $113.5(82.1,144.9)$ & $81.6(56.2,107.0)$ & $120.9(95.5,146.3)$ \\
\hline \multicolumn{5}{|l|}{ Duration of phototherapy (h) } \\
\hline Center A, mean $(95 \% \mathrm{Cl})$ & $95.0(65.4,124.6)$ & $178.4(123.0,233.7)$ & $72.8(50.2,95.4)$ & $146.8(123.3,170.4)$ \\
\hline Center B, mean (95\% Cl) & $38.7(20.2,57.1)$ & $70.3^{\mathrm{a}}(48.6,92.0)$ & $16.4^{\mathrm{a}}(-0.7,33.5)$ & $78.0(61.7,94.3)$ \\
\hline
\end{tabular}

$\mathrm{Cl}$, confidence interval;TSB, total serum bilirubin.

aData are complete (see Tables 1 and 2) for all infants except for two 501-750 g infants and two 750-1,000 g infants from center B for duration of phototherapy measurements.

(intraventricular hemorrhage grades 3 and 4, necrotizing enterocolitis, retinopathy of prematurity, patent ductus arteriosus, bronchopulmonary dysplasia, and late-onset sepsis) did not alter the recorded phototherapy treatment effects.

\section{8- to 22-Month Outcomes}

To further evaluate center differences, post hoc analyses were conducted comparing 18- to 22-month outcomes. Fewer aggressively treated center A infants $(6 / 13$ or $46 \%)$ died or 
Table 4. Mean $(95 \% \mathrm{Cl})$ differences in BAER latencies $(\mathrm{ms})$ between the aggressive and conservative phototherapy treatment conditions

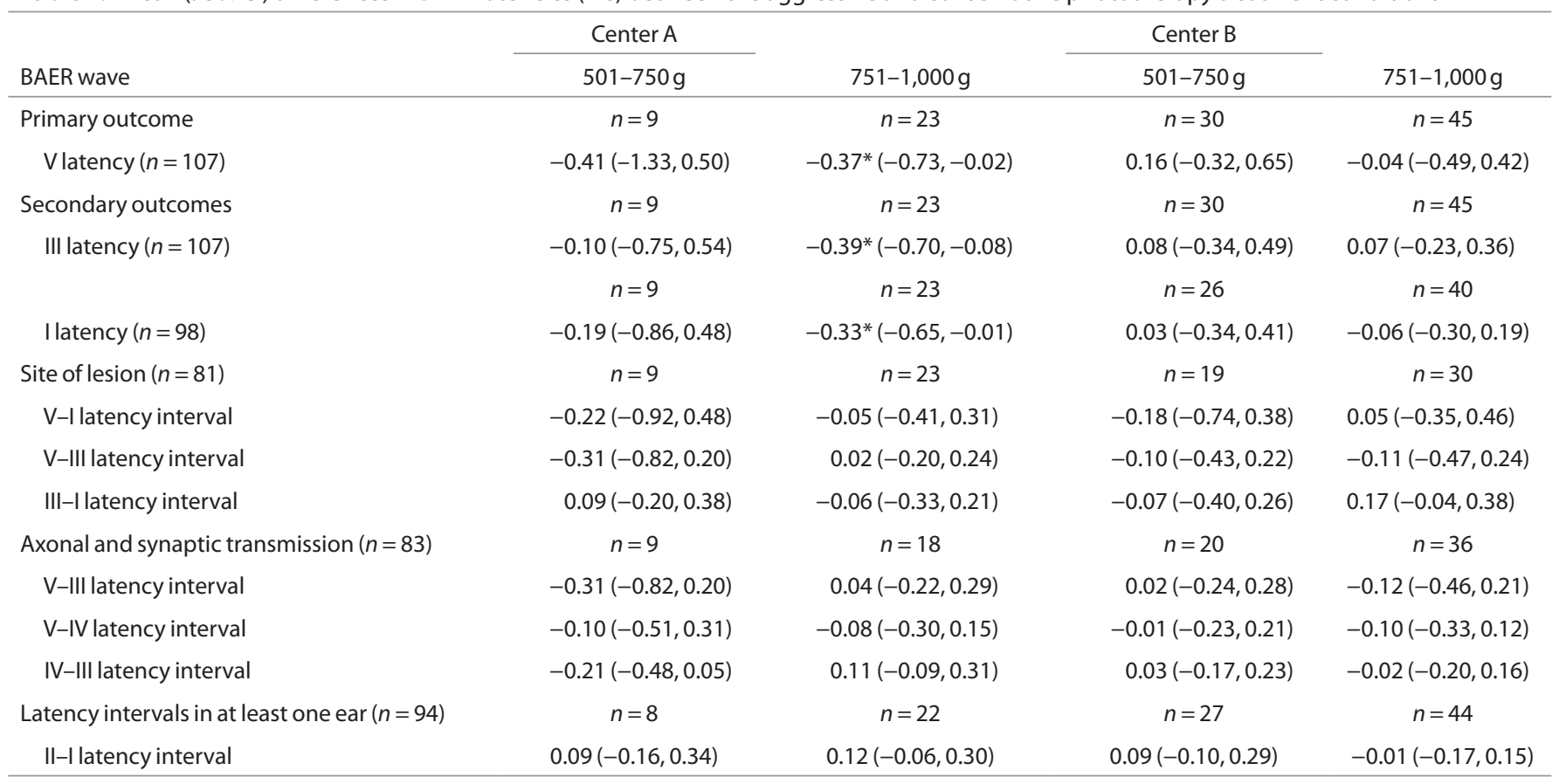

BAER, brainstem auditory evoked responses; $\mathrm{Cl}$, confidence interval. ${ }^{*} \mathrm{P}<0.05$.

had neurodevelopmental impairment at 18-22 months than conservatively treated center A infants (11/18 or $61 \%)$. In contrast, more aggressively treated center B infants $(22 / 34$ or $65 \%)$ died or had neurodevelopmental impairment at 18-22 months than conservatively treated center B infants (18/36 or $50 \%)$. These center differences were also observed for deaths and neurodevelopmental impairment analyzed separately.

Four infants had no scorable BAER waves despite valid recording conditions. All four of these infants were from center $\mathrm{B}$ : one in the $751-1,000 \mathrm{~g}$ stratum receiving conservative phototherapy and the other three in the 501-750 g stratum, one receiving conservative phototherapy and the other two receiving aggressive phototherapy. Three of these infants in this study were the only infants recorded as being deaf at the 18- to 22-month assessment. The other infant with no BAER died before the 18- to 22-month assessment.

\section{Bilirubin Effects}

Each of the four bilirubin variables was positively correlated with the BAER V, III, and I latencies but not the interwave intervals for the 751-1,000 g center A infants in the aggressive treatment arm. The regression coefficients were of similar magnitude for all three waves for these infants (V, III, and I latencies are highly correlated) and 8/12 (three waves by four bilirubin variables) were statistically significant at the 0.05 level. For wave V, there was a $0.05-\mathrm{ms}(95 \% \mathrm{CI}=-0.05,0.16)$ latency increase for every $\mathrm{mg} / \mathrm{dl}$ increase in 14-day TSB average, a $0.06-\mathrm{ms}(95 \% \mathrm{CI}=-0.04,0.16)$ latency increase for every $\mathrm{mg} / \mathrm{dl}$ increase in peak TSB over the 14-day treatment interval, a $0.09-\mathrm{ms}(95 \% \mathrm{CI}=0.01,0.18)$ latency increase for every $24-\mathrm{h}$ increase in the age at peak TSB, and a $0.15-\mathrm{ms}$ ( $95 \%$ $\mathrm{CI}=0.02,0.27)$ latency increase for every 24 -h increase in the duration of phototherapy. For the 751-1,000 g center A infants, $11 / 12$ correlations were positive in the conservative treatment arm, although only one was significant at the 0.05 level. There were only three significant positive correlations for infants in the 72 other combinations of center, birth-weight stratum, and phototherapy treatment.

\section{DISCUSSION}

Weeks after aggressive phototherapy that only modestly reduced bilirubin exposures (see Table 3); BAER wave V, III, and I latencies were significantly shorter for 751-1,000 g birth-weight center A newborns (see Table 4). Similar nonsignificant trends were recorded for the 501-750 g birthweight infants at center A. Neuronal transmission in the brainstem and axonal and synaptic transmission did not differ in the aggressive and conservative treatment conditions. We do not know whether these effects persist past the newborn period, although aggressive phototherapy was associated with a significant reduction in hearing impairment in the parent trial (type of loss did not differ in the aggressive and conservatively treated infants) (3).

The lesion site responsible for altered BAER latencies in center A could be anywhere in the auditory pathway peripheral to the distal auditory nerve. Prior to testing, the ear canal was visually examined to ensure that obstruction of the outer ear canal did not affect the BAER recordings. Middle ear and cochlear testing were not conducted and cannot be ruled out as lesion sites. These results do not support the hypothesis that 
bilirubin at these levels of exposure alters neural transmission in the brainstem of ELBW newborns, contrasting with results from laboratory studies on animal models and observational studies largely on late-preterm and term newborns with very different bilirubin exposures (4).

Discrepancies between the results we report and studies in the literature may be explained by differences in the newborns sampled, bilirubin exposures, time of BAER assessment, medical care, and study methods. This is the only study randomly allocating ELBW newborns to treatment conditions resulting in different bilirubin exposures. Random allocation minimizes imbalances in measured and unmeasured confounders, increasing the likelihood of evaluating the true relationship between bilirubin exposure and BAER. We conducted adjusted analyses to further confirm the likelihood of exchangeable treatment groups. Our study was powered to detect a meaningful effect size, enrolled all eligible newborns, minimized missing data, and standardized and blinded measurements and analyses to reduce bias. Secondary studies of clinical trials provide randomly allocated group comparisons to research otherwise largely limited to observational studies.

Animal models of hyperbilirubinemia have been used to evaluate neurotoxic effects in the auditory brainstem $(4,6,7)$. The animal models developed may not be appropriate for the phototherapy-induced effects reported in this study because neural transmission in the brainstem was not altered.

No significant phototherapy treatment effects were recorded for center B infants. Differences in newborn baseline characteristics, neurodevelopmental outcomes, phototherapy, and BAER methodology may explain the center differences in the treatment effects we recorded. Adjusting for measured demographic and clinical differences did not account for center differences, although unmeasured confounders may explain center differences. Aggressively treated infants at center A but not center B were more likely to survive without neurodevelopmental impairment. This result is consistent with better outcomes for infants from center A but at odds with no effect on neural transmission in the brainstem.

The duration of phototherapy was longer and the 14-day average and peak TSB levels higher at center A than center B. The 14-day average and peak TSB levels in the center B conservative treatment conditions were comparable to the center A aggressive treatment condition levels. Bilirubin exposures in the center B conservative treatment condition may not have been high enough or long enough to prolong BAERs at 35 weeks PMA. In addition, the fiber-optic blankets used in center $\mathrm{A}$ and the phototherapy lights used in center B may change bilirubin into different photoisomers $(8,9)$ with different effects on the auditory pathway.

The vagaries of sampling may also explain center differences. We cannot determine from this study whether rejecting the null hypothesis of no association between aggressive phototherapy and BAER latencies based on center A results commits a type I error or whether failing to reject the null hypothesis based on center B results commits a type II error.

We report that reducing bilirubin exposures in some ELBW infants by aggressive use of phototherapy reduced BAER latencies without altering brainstem transmission. We also report that BAERs of ELBW newborns with slightly different exposures and clinical characteristics were not differentially affected by more aggressive phototherapy. Given the complexity of the biology, it should not be surprising that bilirubin's effects are variable depending on bilirubin exposures, the newborns evaluated and their clinical conditions, and study methodologies. A more complete understanding of the effects of bilirubin on newborns requires rigorous research methodologies and a better understanding of bilirubin metabolism at different stages of development and in different clinical conditions.

\section{METHODS}

\section{Trial Design}

This study was a two-center, stratified (center and 501-750g/ 751-1,000 g birth-weight strata), parallel-group randomized trial. Eligible infants were randomly allocated (1:1 ratio) to the conservative or aggressive phototherapy treatment conditions within center and birth-weight stratum. See Morris et al. for details concerning study design and methodology (3).

\section{Participants}

All 501-1,000g birth-weight infants participating in the NICHD Neonatal Research Network Phototherapy trial from two network centers who were born within defined enrollment periods were eligible for this substudy. Enrollment periods for center A (infants who were 35 weeks PMA after 17 January 2003 but born no later than 1 September 2004) and center B (infants who were 35 weeks PMA after 11 August 2003 but born no later than 3 February 2005) depended on demonstrated adherence to the substudy protocol. Consent for this substudy was nested within the parent-study consent, approved by each center's internal review board, and obtained from each study newborn's parent or custodian.

\section{Intervention}

Protocol-stipulated phototherapy use was for the first 14 postnatal days. Phototherapy was administered almost entirely by fiber-optic blankets at center A and entirely by phototherapy lights at center B. Bilirubin levels were measured by TSB averaged over the 14 measurement days, the peak TSB during those 14 days, and the age at that peak TSB. The total duration of phototherapy was also measured.

\section{BAER Recording Protocol}

Study infants were tested during their 35th week PMA or within 3 days of discharge if discharged prior to 35 weeks. If testing could not be completed according to protocol because the baby was not in a testable state, the technical aspects of the recordings were unacceptable, or the medical staff refused to allow testing due to the unstable medical condition of the infant, the infant was tested as soon as possible to obtain an acceptable recording.

Each ear was assessed separately by presenting $80-\mathrm{dB}$ normal hearing level (nHL), 100- $\mu$ s clicks at a rate of 20.1 clicks per second. The BAER responses were differential recordings from scalp electrodes pasted on the forehead at midline (noninverting electrode), the ipsilateral mastoid (inverting electrode), and the contralateral mastoid (the ground). The responses were amplified 100,000 times, filtered from $100 \mathrm{~Hz}$ to $3 \mathrm{kHz}$, and averaged for 2,000 repetitions. Four averages were recorded from each ear, the first three to rarefaction clicks 


\section{Phototherapy and the BAER}

and the last to a condensation click. By adding rarefaction and condensation responses, polarity-sensitive cochlear responses cancel while polarity-insensitive neural responses add, providing a way to distinguish cochlear from neural responses (critical for the diagnosis of auditory neuropathy). No cases of auditory neuropathy were identified. The outcome measurements were made from the two best responses to the rarefaction clicks.

The electrode impedances were required to be $<10 \mathrm{k} \Omega$ and were measured before and after testing each ear. The noise levels in the room, the infant's testability (state, movement, etc.), and technical adequacy (electrical artifact) were assessed on 5-point Likert scales by the recording audiologists.

\section{Scoring the BAER Waveforms}

BAERs record sound-induced neural activity from successive generators along the auditory pathway. The generators for waves I, II, III, IV, and $\mathrm{V}$ are the distal and proximal auditory nerves, the cochlear nucleus, the superior olive, and the lateral lemniscus, respectively (10). The primary outcome was wave V latency at 35 weeks PMA. Because the primary site of bilirubin-induced toxicity was hypothesized to be the auditory brainstem, the V-I interwave interval might have been selected as the primary outcome. It was not selected because, prior to the study, we anticipated the difficulty in recording wave I reliably from sick infants in neonatal intensive care units.

Secondary outcomes included wave I and III latencies. In addition, secondary analyses were conducted on infants with reliably scored waves I, III, and V in both ears $(n=81$ with complete data of the 111 infants tested) to evaluate site-of-lesion effects. The V-I, V-III, and III-I interwave latency intervals measure transmission times between the respective generators of those waves. A second set of secondary analyses were conducted on infants with reliably scored waves III, IV, and $\mathrm{V}$ in both ears ( $n=83$ with complete data) to distinguish axonal transmission from transmission involving a synapse. Ponton and Eggermont hypothesized that the V-III and III-I interwave latency intervals measure both axonal and synaptic transmission, whereas the IV-III and II-I intervals measure only axonal and not synaptic transmission (5). The II-I latency interval was not included in our secondary analyses a priori because we anticipated a small number of newborns with reliably scored II-I intervals. We recorded II-I intervals reliably in both ears for 55 infants, although they could be scored in at least one ear for 94 infants.

Data files, de-identified with the center number and network ID for each infant, were electronically transferred to the NICHD Neonatal Network Data Center (RTI International) in North Carolina. At the end of the study, separate data files for independent scoring were provided to the two scorers (M.S.O, R.E.L) with more than 20 years experience, who scored all the BAER study waveforms. "Reliable" measurements were identified as within $0.20 \mathrm{~ms}$ of each other. The means of the reliable measurements from the two scorers were used as the data analyzed for this study. If a measurement was not reliable, the two scorers reviewed the discrepancy and generated a consensus measurement used in the analyses.

\section{Sample Size}

This study was powered a priori to detect an effect size $(0.25$-ms reduction in wave $\mathrm{V}$ latency with $\mathrm{SD}=0.50 \mathrm{~ms}$ ) comparable to a decrease in wave $\mathrm{V}$ latency in preterm newborns over a 2-week interval (11). Detecting that effect size with a type I error of 0.05 and a type II error of 0.20 required 64 infants in each treatment condition. Although 174 infants were enrolled, only 111 infants (51 in the aggressive and 60 in the conservative treatment conditions) were tested according to protocol with interpretable waveforms. Most of the "missed" infants $(57 / 63=$ 90.5\%) died before 35 weeks. Given the type I (0.05) and II (0.20) error rates assumed and 111 infants sampled, we could expect to reliably detect a $0.27-\mathrm{ms}$ rather than a $0.25-\mathrm{ms}$ reduction in wave $\mathrm{V}$ latency. We had $74 \%$ power to detect the 0.25 -ms reduction.

\section{Randomization}

Newborns were stratified by center and birth weight (501-750g and $751-1,000 \mathrm{~g})$ and then randomized to one of the two treatment conditions by calling a centralized computer system at RTI International.

\section{Blinding}

The study personnel recording the BAERs did not know the treatment assignments. The scoring of the BAER waveforms was also done blinded to treatment assignment.

\section{Statistical Methods}

The analyses were updated prior to analyzing the data to be consistent with the analyses of the NICHD Neonatal Research Network parent trial (3). That trial was designed to evaluate two different phototherapy regimens. In addition, the parent trial evaluated whether birth weight and also center (unpublished analyses) moderated treatment effects. Analyses were intention to treat. $P$ values $<0.05$ were considered statistically significant for all analyses, with no adjustment for multiple comparisons. Analyses were conducted using Stata (version 10.1, College Station, TX).

Linear regression models including phototherapy treatment condition, the two stratification variables (center and birth-weight category), all two-way interactions, and the three-way interaction were calculated to evaluate treatment differences in BAER outcomes. A clustered sandwich estimator was used to estimate standard errors for the BAER outcomes because of the correlation between measurements from the two ears of each newborn (12). The referents for the independent variables in these models were the conservative phototherapy treatment arm, center $\mathrm{A}$, and the $751-1,000 \mathrm{~g}$ birth-weight stratum. The interaction terms indicated whether the effect of aggressive phototherapy was moderated by center and birth-weight stratum as evaluated in the NICHD Neonatal Research Network trial and specified prior to conducting the analyses.

Additional linear regression models were calculated to adjust for variables that might confound phototherapy treatment effects because of an imbalance in those variables despite random allocation. We evaluated the baseline and demographic variables evaluated in the NICHD Neonatal Research Network trial as potential confounders by adding each baseline and demographic variable to the linear regression models described above. In addition, a forward stepwise procedure was calculated to evaluate multiple confounders simultaneously $(P=0.20$ to enter).

In four infants, no BAERs could be identified, consistent with profound hearing losses. Those responses were censored and Tobit regressions were calculated to include the censored responses in the analyses (13). The results of those analyses were similar to the results of the linear regressions described above and are not reported.

The effects of bilirubin levels (average levels for the first 14 days after initiation of therapy, the peak bilirubin levels during those first 14 days, and the age at the peak measurement) and the duration of phototherapy on the BAER were evaluated by linear regression models including main effects for each bilirubin variable, the two stratification variables (center and birth-weight category), all two-way interactions, and the three-way interaction. A clustered sandwich estimator was used to estimate standard errors accounting for the correlation between measurements from the two ears. In addition, simple regressions (BAER variable regressed on bilirubin variable) were calculated for infants from the eight combinations of center, birth-weight category, and phototherapy condition.

\section{ACKNOWLEDGMENTS}

We are indebted to our medical and nursing colleagues and the infants and their parents who agreed to take part in this study. The following institutions and investigators, in addition to those listed as authors, participated in this study. Alan Jobe, University of Cincinnati (NRN Chair, 2001-2006). Alpert Medical School of Brown University and Women \& Infants Hospital of Rhode Island (U10 HD27904). Cincinnati Children's 


\section{Articles | Lasky et al.}

Hospital Medical Center University Hospital and Good Samaritan Hospital (U10 HD27853, M01 RR8084). Linda L. Wright, Elizabeth M. McClure: Eunice Kennedy Shriver National Institute of Child Health and Human Development. W. Kenneth Poole, Betty Hastings, John C. Langer, Rebecca L. Perritt, Qing Yao, Carolyn Petrie Huitema, Sarah Taylor, and Kristin Zaterka-Baxter: RTI International (U10 HD36790). Stanford University Dominican Hospital, El Camino Hospital, and Lucile Packard Children's Hospital (U10 HD27880, M01 RR70). University of Rochester Medical Center Golisano Children's Hospital (U10 HD40521, M01 RR44). Kathleen A. Kennedy, Esther G. Akpa, Patty A. Cluff, Anna E. Lis, Claudia Y. Franco, Maegan Currence, Nora I. Alaniz, and Patti L. Pierce Tate: University of Texas Health Science Center at Houston, Medical School, Children's Memorial Hermann Hospital, and Lyndon B. Johnson General Hospital (CCTS KL2 RR24149, CCTS UL1 RR24148, U10 HD21373). Wake Forest University Baptist Medical Center, Brenner Children's Hospital, and Forsyth Medical Center (U10 HD40498, M01 RR7122). Rebecca Bara and Geraldine Muran: Wayne State University Hutzel Women's Hospital and Children's Hospital of Michigan (U10 HD21385).

\section{STATEMENT OF FINANCIAL SUPPORT}

The National Institutes of Health and the Eunice Kennedy Shriver National Institute of Child Health and Human Development provided grant support for the Neonatal Research Network's Phototherapy Study (U10 HD21373, U10 HD21385, U10 HD27853, U10 HD27880, U10 HD27904, U10 HD36790, U10 HD40498, U10 HD40521, KL2 RR24149, M01 RR44, M01 RR70, M01 RR7122, M01 RR8084, UL1 RR24148).

\section{REFERENCES}

1. Scheidt PC, Bryla DA, Nelson KB, Hirtz DG, Hoffman HJ. Phototherapy for neonatal hyperbilirubinemia: six-year follow-up of the National Institute of Child Health and Human Development clinical trial. Pediatrics 1990;85:455-63.
2. Brown AK, Kim MH, Wu PY, Bryla DA. Efficacy of phototherapy in prevention and management of neonatal hyperbilirubinemia. Pediatrics 1985;75(2 Pt 2):393-400.

3. Morris BH, Oh W, Tyson JE, et al.; NICHD Neonatal Research Network. Aggressive vs. conservative phototherapy for infants with extremely low birth weight. N Engl J Med 2008;359:1885-96.

4. Shapiro SM, Nakamura H. Bilirubin and the auditory system. J Perinatol 2001;21 Suppl 1:S52-5; discussion S59-62.

5. Ponton CW, Moore JK, Eggermont JJ. Auditory brain stem response generation by parallel pathways: differential maturation of axonal conduction time and synaptic transmission. Ear Hear 1996;17:402-10.

6. Kuriyama M, Konishi Y, Sudo M. Auditory brainstem response in hyperbilirubinemic rat: Part I. Biol Neonate 1990;58:32-40.

7. Haustein MD, Read DJ, Steinert JR, Pilati N, Dinsdale D, Forsythe ID. Acute hyperbilirubinaemia induces presynaptic neurodegeneration at a central glutamatergic synapse. J Physiol (Lond) 2010;588(Pt 23):4683-93.

8. Hansen TW. Twists and turns in phototherapy for neonatal jaundice. Acta Paediatr 2010;99:1117-8.

9. Mreihil K, McDonagh AF, Nakstad B, Hansen TW. Early isomerization of bilirubin in phototherapy of neonatal jaundice. Pediatr Res 2010;67:656-9.

10. Moller AR, Jannetta PJ. Neural generators of the auditory brainstem response. In: Jacobson JT (ed), The Auditory Brainstem Response. San Diego: College-Hill Press, 1985:13-31.

11. Lasky RE. A developmental study on the effect of stimulus rate on the auditory evoked brain-stem response. Electroencephalogr Clin Neurophysiol 1984;59:411-9.

12. Rogers WH. Regression standard errors in clustered samples. Stata Tech Bull 1993;13:19-23.

13. Tobin J. Estimation of relationships for limited dependent variables. Econometrica 1958;26:24-36. 